\title{
Contaminated Food and Water
}

\section{CONTAMINATED FOOD}

\section{General}

Food Susceptibility. Stored, transported, and prepared food is susceptible to chemical contamination throughout the Theatre of Operation (TO). Planning for any battle or operation must include food protection from contamination, food contamination detection and contaminated food disposition (decontaminate or destroy).

Countermeasures. There are three primary countermeasures to overcome or reduce the chemical hazard to food:

- contamination avoidance.

- chemical agent detection.

- chemical agent decontamination.

Priorities. The priorities for conducting chemical countermeasures are:

- Contamination avoidance. Contamination avoidance includes using natural and fabricated barriers to prevent, or significantly reduce the spread of contamination. Additionally, specific procedures for entry and exit between contaminated and uncontaminated areas to reduce the potential for spreading contamination should be used. Use of these barriers and procedures may reduce the subsequent need for detection and decontamination.

- Detection, measurement, and identification. These activities are essential for determining the presence, extent, and nature of chemical contamination. This information is essential in identifying the existence of uncontaminated supplies, or decontamination requirements.

- Decontamination. Decontamination removes the contaminant and provides food that is safe for consumption. Decontamination efforts require an extensive amount of labour, time, and supplies. The use of hasty decontamination is emphasised, i.e. decontaminate just enough to sustain operations and keep fighting, rather than to make a contamination-free environment. Normally, decontamination efforts will be limited to the packaging and packing materials. Food decontamination will only occur in critical situations where other food supplies are not available. Most decontamination is performed in or very near the Area of Operation (AO). Before beginning decontamination procedures, exposed food items should be divided into groups based on protection of item at time of exposure. These groups establish priorities based on ease of decontamination and the ability to monitor the food.

- Group I-Canned or packaged items exposed only to a chemical agent vapour.

- Group II-Canned or packaged items that are contaminated on the outside with a liquid chemical agent.

- Group III-Unpacked or poorly packaged items that have been exposed to a chemical agent.

- Group IV-Food contaminated through the food chain.

\section{Protection of Food from \\ Contamination}

An adequate defensive posture for a chemical attack will also protect food against biological contamination and radiation fallout.

\section{Operational Rations}

Operational rations include (but are not limited to) individual rations, group meals that are ready-to-eat, survival rations, unit rations, and medical rations.

Packaging materials and storage methods normally protect these rations. The packaging and packing of operational rations protect the contents from deterioration. As a result, the contents are protected from moisture, to include chemical agent liquids and vapours. Operational rations delivered to an $\mathrm{AO}$ will usually have increased levels of packaging and/or packing protection. Operational rations are substantially protected while contained in the shipping cases, especially if protected with an overlay of fibreboard, shrink wrap, or film wrap.

Enclosed storage should be used whenever possible. Refrigerated warehouses, cold storage rooms, and even prefabricated refrigerators and trailers provide excellent protection. Underground shelters, caves, and tunnels that can be made airtight provide maximum chemical protection. Buildings provide protection depending on how well they can be closed and sealed. The basement of a building is a good storage place. However, keep in mind that chemical vapours tend to seek out low-lying areas. Storing rations indoors will protect them from liquid droplet unless the building is 
damaged by an attack. Complete protection against chemical vapours is only offered by airtight closed spaces like cold storage facilities.

Chemical protective measures are to be integrated into daily logistical operation to avoid the contamination of operational rations. Maximum use should be made of alarm and detection equipment, overhead shelter, shielding materials, and protective covers. Back up stocks of operational rations should be dispersed to minimise the risk of destruction or contamination.

A protective cover or similar equipment will assist. The protective cover should be discarded and replaced upon becoming contaminated; it reduces overall decontamination requirements and it improves the survivability of supplies and equipment. The protective cover provides 24-hour protection against liquid chemical contamination. Detection paper used on the protective cover will rapidly identify a contaminated cover.

\section{Bulk and Fresh Foods}

Field expedient or improvised storage may be the only choice available under high risk conditions. Expedient storage for food supplies may be a natural or man-made depression lined to protect contents against moisture, and then covered with earth and sod. The earth gives good protection against all forms of chemical or biological contamination and nuclear fallout.

Foods should only be stored outdoors or in partially protected areas when absolutely necessary. Only cases of foods packed in cans, bottles, or airtight foil or film wraps, and foods packed in sealed boxes or multilayered wrappings can be subjected to exposed storage. Open sheds, temporary roofing, or tents provide partial protection. When subsistence must be stored in the open, give as much protection as possible. Protection material may include protective covers (tarpaulins), tarpaulin sheds, or any other available covering such as plastic sheeting. Tarpaulins and other treated or waterproof coverings do not prevent contamination by chemical vapours, but they do reduce contamination from liquid agents. Canvas will keep out more than $95 \%$ of liquid contamination for a short period of time after the attack. The canvas must be removed soon after the attack to prevent the agent from seeping through onto the subsistence; placement of spacers between the covering and the food will greatly reduce this problem. Even the thinnest material will offer some protection and is better than nothing at all. Therefore, food supplies must be covered by whatever material is available.

\section{Chemical Contamination}

Contamination of foodstuffs by a chemical agent may occur at any point on the battlefield. This contact may render the food unpalatable also. In many cases, decontamination is difficult and so emphasis must be placed on protection. Food supplies should be covered at all times. Unprotected food, forage, and grain supplies may be so contaminated that their consumption will produce gastrointestinal irritation, or systemic poisoning. Nerve agents, vesicants, and arsenicals are the most dangerous. Field concentrations of phosgene, hydrocyanic acid, irritants, and smokes will seldom be high enough to cause serious food contamination. The effect of CK (cyanogen cyanide) on food is not known. As a precaution, foods exposed to CK should be considered toxic.

The effects of chemical agents on food depend on the nature of the agent and the type of the food. The extent to which chemical agents penetrate food also depends on the amount, form of dispersal (liquid [droplet size], or vapour) and duration of exposure. Nerve agents and mustard will penetrate deeply into unprotected fatty foods and will readily penetrate granular products such as grain and sugar. Liquid food products can be completely contaminated. Arsenicals readily hydrolyse to poisonous arsenical oxides in some foods. Foods can be divided into three categories based on their water content, fat content, and crystalline structure:

(1) Foods having a high water content, a low fat content, and/or a crystalline structure (fresh vegetables, fruits, sugar, salt, and eggs), will absorb mustard and nerve agents, either as a liquid or as a vapour. Nerve agents hydrolyse slowly.

(2) Foods having a low fat content and an irregular (amorphous) structure (flour, bread, grain, rice, cereals, dried fruits, dried vegetables, tea, coffee, peas, and beans), readily absorb mustard and nerve agent in liquid form. As a vapour, these agents are absorbed to some extent, but are easily removed by airing.

(3) Foods having a low water content and a high fat content such as butter, fat, fatty oils, ham, cheese, milk, bacon, fatty meat, and fish, absorb mustard and nerve agents such that removal of the agents is virtually impossible.

Chemical agents can be physically and chemically absorbed into food. In addition to the toxic effect, they often adversely affect taste, smell and the appearance of the food. However, chemical agents can cause the food to become very toxic without causing any other changes in the food. Since food can be contaminated without any outward change in appearance, the possibility of contamination must be assumed in a chemical agent environment. 


\section{Detection}

Currently, a field method for detecting chemical agent contamination in food is not available. Contamination is not always spread evenly throughout food; this makes it impossible to take a single sample and determine the presence or absence of chemical agents in the entire lot. Additionally, standardised laboratory tests have not been developed for determining levels of chemical agents in food. Until a specific method to detect chemical agents in food is available, reliance will have to be made upon determination of contamination, or lack thereof, on the packaging material, the integrity of the packaging material, the protective qualities of the packaging material and the penetration characteristics of the suspected chemical agents.

Food may become toxic without any change in outward appearance. Never taste or smell food to determine if contamination is present.

Veterinary and subsistence units have equipment available to detect chemical agents in the field. All subsistence in a chemical attack area are considered contaminated until a survey can be conducted, preferably by veterinary and chemical personnel. Personnel must be at highest individual protective equipment level while conducting the survey. The initial portion of the survey should focus on the adequacy of the storage facility and other protective measures in preventing chemical agent contact with subsistence items. The area surrounding the storage facility should be examined for the presence of animals, rodents, birds, and arthropods acting unusually, or dead in unusual numbers. If animals are present and assistance is required in identifying the chemical agent, specimens can be collected and submitted for laboratory examination. Damage such as broken windows, holes, or loss of structural integrity of the storage facility should be noted. This information, combined with knowledge of the agent form (liquid or vapour), type of agent (which will indicate the degree of persistency), and approximate time of attack will provide a risk assessment. Liquid agents should not significantly penetrate an intact facility, but may produce vapour contamination by off-gassing.

\section{CONTAMINATED WATER}

\section{General}

Water supplies in areas with chemical contamination and in surface water supplied by run-off from such areas will most likely be contaminated. The contamination of water, whether intentional or inadvertent, may reach concentrations that will produce casualties. By special methods of analysis, the presence of contamination can be determined. Treatment of contaminated water requires chemicals and equipment that are only available to water purification units; individuals or units should not attempt to treat their water. Decontamination of water is only undertaken when uncontaminated sources are not available; then ONLY with the approval of the medical authority.

\section{Detection of Contamination in Water}

The Chemical Agent Water Testing Kit provides a rapid field test to detect chemical agent contamination in water. The test must be conducted before the water is treated with chlorine; the chlorine will affect the accuracy of the test for chemical agents.

\section{Procedures on Discovery of Contamination in Water}

When contamination is discovered the following actions are taken:

- the water source should be marked using the standard contamination markers, and personnel must not consume the water until approved.

- the commander should be notified that the water source is contaminated and unfit for drinking, food preparation, and personal hygiene.

- the commander should establish safeguards to prevent personnel from using the contaminated water supply.

- an alternative source of uncontaminated water must be sought and used.

The primary source for obtaining water is from water production and distribution points. Other sources are considered only when water production and distribution facilities are not available. Alternative sources that may be considered include:

- ground water source, which is least likely to be contaminated.

- local fixed facility water supplies.

- movement to another location to obtain an uncontaminated water source, when the tactical situation permits.

Contaminated water must not be used until it has been treated by water production and distribution points units or other equally capable water purification units and approved for use by the medical authority.

\section{Treatment of Contaminated Water}

Contaminated water requires additional equipment and supplies to remove the contamination. Water purification and distribution units must be equipped to perform these duties. 